\title{
The Nature of Weather and Climate Impacts in the Energy Sector
}

\author{
David J. Brayshaw
}

Abstract The power sector's meteorological information needs are diverse and cover many different distinct applications and users. Recognising this diversity, it is important to understand the general nature of how weather and climate influence the energy sector and the implications they have for quantitative impact modelling. Using conceptual examples and illustrations from recent research, this chapter argues that the traditional 'transfer function' approach that is common to many industrial applications of weather and climate science-whereby weather can be directly mapped to an energy impact - is inadequate for many important power system applications (such as price forecasting and system operations and planning). The chapter concludes by arguing that a deeper understanding of how meteorological impacts in the energy sector are modelled is required.

Keywords Climate variability $\bullet$ Energy sector $\bullet$ Power $\bullet$ Impact modelling - Weather impact $\bullet$ Meteorological information • Power demand

D.J. Brayshaw $(\bowtie)$

Department of Meteorology, University of Reading, Reading, UK

National Centre for Atmospheric Science, Reading, UK

(C) The Author(s) 2018

A. Troccoli (ed.), Weather \& Climate Services for the Energy Industry, https://doi.org/10.1007/978-3-319-68418-5_11 


\section{Weather and Climate Impacts in the Energy Sector}

The power sector's meteorological information needs are diverse. On the one hand, Transmission System Operators (TSOs) may be concerned with detailed geographical forecasts of wind power and demand at relatively short lead times (hours or days ahead) for the operational management of the power grid. This contrasts, for example, with long-term investors in infrastructure and system planners who require a longer view of system resilience (years to decades), and energy traders or maintenance planners seeking to position themselves for the coming weeks or seasons.

A common theme, however, is the need for a series of conversions to transform meteorological information into an actionable decision. The three steps in Fig. 11.1 can typically be recognised.

Chapter 6 discussed the first step in this process at length, and understanding user needs and preferences is discussed elsewhere in other chapters of this book (Chaps. 1, 3, 4 and 5). Here, the focus is on the general process of modelling energy system impacts using meteorological data from numerical simulations, illustrated with selected examples (i.e., Impact Simulation). It is, however, noted that user preferences-once elicited and expressed quantitatively - can be thought of as a conversion of a physical impact (in terms of MWh, prices, loss of load) into a 'utility' (a numerical expression of the user's preferences). To some extent, they can therefore be considered as direct extensions of the impact models discussed below.

It is helpful to identify three distinct levels of complexity in weatherand climate-impact modelling, as illustrated in Fig. 11.2.

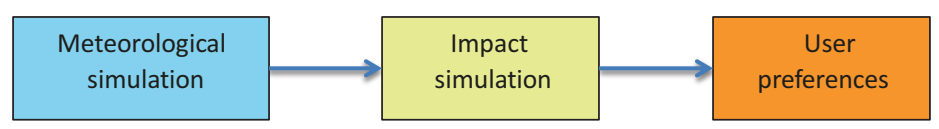

Fig. 11.1 The process of converting meteorological data into actionable information

Fig. 11.2 Levels of impact complexity

Single impact

Compound impact

Complex impact 

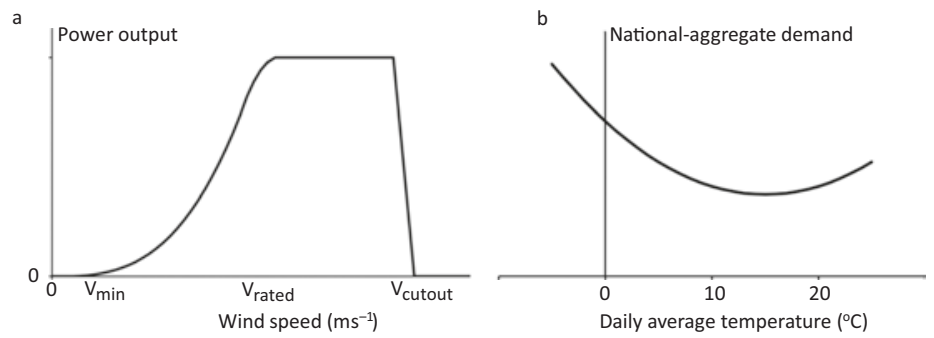

Fig. 11.3 Simple examples of idealised transfer functions used to convert meteorological quantities into estimates of power system properties: (a) an idealised wind power curve based on Brayshaw et al. (2011); (b) a simplified demand model based on Bloomfield et al. (2016). In each example here, the transfer function is shown to depend only on a single meteorological variable for simplicity but in general they may incorporate many input variables. Additional dependencies may be meteorological (e.g., wind direction for wind power, cloud cover for demand) or non-meteorological (e.g., day-of-week for demand), and include stochastic 'noise' to simulate the error and uncertainty in the transfer function

The simplest level, commonly referred to as 'point forecasting', may be defined as the response of a single energy system component to a set of meteorological drivers for which a transfer function can be written. Typical examples might include predicting wind power output for a particular turbine, farm or country, or forecasting power demand over a particular geographical region (Fig. 11.3). The key aspect is that it is possible to write (or otherwise estimate in at least an approximate form) a function, $f$, which converts a set of meteorological variables, $\{m\}$, into the energy system property of interest $E$ :

$$
E=f(\{m\})
$$

The transfer function may be either physically or empirically derived, may be non-linear, many-to-one or probabilistic. Typical examples include electricity demand models (Thornton et al. 2016; Taylor and Buizza 2003), wind power production models (Dunning et al. 2015; Cannon et al. 2015) and damage models (McColl et al. 2012).

A more complex form of impact occurs when the simultaneous influence of meteorology on several different components of an energy system becomes an important part of the impact. In this case, a transfer function 
exists for each component, but the impact is perceived through a combination of those components (often referred to as a 'compound impact'). An example is the residual power load of national power systems (i.e., demand net renewables), which depends on demand, solar and wind, each of which has a different sensitivity to weather.

In the simplest case, the system can be considered as a set of noninteracting energy system components, $\{E\}$, and may be written:

$$
S=L(\{E\})
$$

where $L$ is a mapping of the set of energy system components $\{\mathrm{E}\}$ to a particular system-wide property of interest, $S$.

An example ${ }^{1}$ of this is the 'merit order' model $^{2}$ of UK wholesale power price, exploring the extent to which month-ahead forecasts could be beneficial to energy-trading and risk management (Fig. 11.4a; see Lynch et al. 2014; Lynch 2016). In this example, daily ensemble forecasts of UK wind power and national total power demand are created from the European Centre for Medium-Range Weather Forecasts (ECMWF) system for several weeks in advance, and the 'residual demand' calculated. ${ }^{3}$ The residual demand is assumed to be met by a mixture of coal and gas generation, preferentially utilising the cheapest marginal cost generators first (i.e., those bidding to produce power at the lowest price), with the wholesale power price being determined by the most expensive generation unit required to operate (Fig. 11.4b). The use of sub-seasonal weather forecasts three to four weeks ahead was shown to offer an improvement over standard industry practice for some-though not all-trading applications. This work therefore emphasised both the potential benefits of longer-range meteorological forecasts for energy, but also the need for careful evaluation of the forecast's performance in the context for which it is being used.

In both 'point impact' and 'compound impact' problems, the meteorological state is assumed to map directly to that of the impacted system (via a transfer function or set of transfer functions) and, although the mapping may be complicated, it is only dependent on the current meteorological state. This assumption does not hold, however, in many energy system planning and operations problems (e.g., 'optimal power flow' or 'unit commitment'). In problems of this type, there are potentially complex connections in time and space between different energy system components and to forecast the state of the impacted system accurately requires 

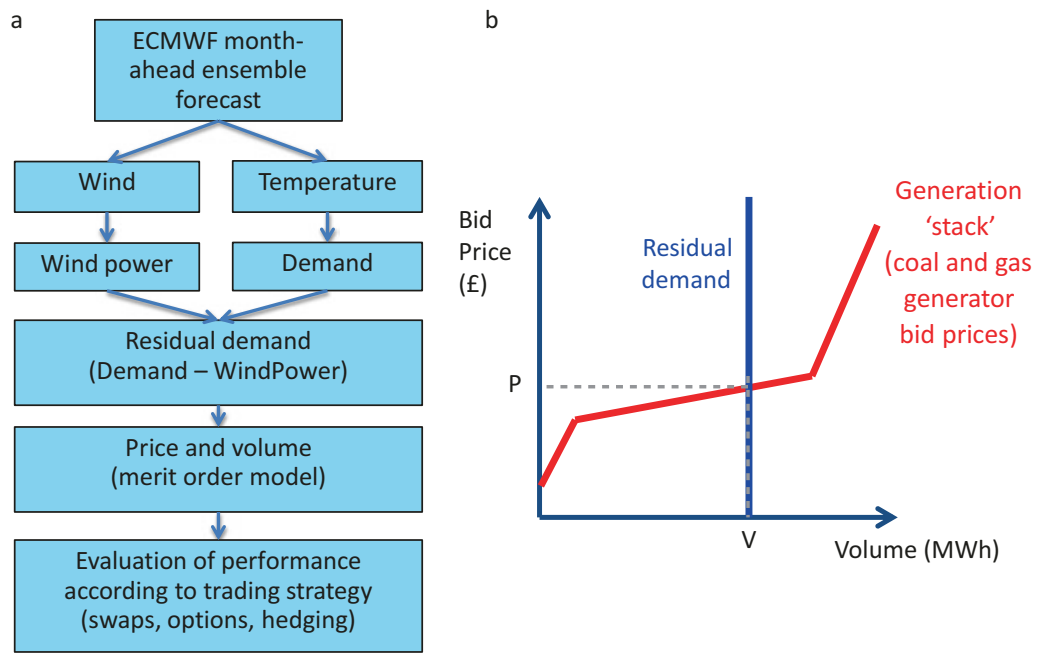

Fig. 11.4 An illustration of energy price forecasting using meteorological inputs following Lynch (2016) and Lynch et al. (2014). (a) A flow chart illustrating the process through which the forecast is made and evaluated. (b) A schematic of the 'merit order model'. In (b), the red curve indicates the relationship between supply and price (more expensive power stations are willing to produce as price rises, hence a positive relationship between volume and price). The blue curve indicates the relationship between demand and price (the demand for power decreases with price, but here is assumed to be perfectly price-insensitive). The intersection of the two curves sets the wholesale price and volume of power produced by the market. The qualitative shape of the supply curve produced by the two-generation type model (as fitted by Lynch (2016) to observed price data using an Ensemble Kalman filter) is indicated in (b). Lynch (2016) went on to demonstrate that the ECMWF-forecast based process outlined in (a) was able to significantly outperform equivalent forecasts using purely historical weather observations for each of wind power, demand and price (evaluated over the period December 2010February 2014, at a 99\% statistical confidence level). ECMWF stands for European Centre for Medium-Range Weather Forecasts

knowledge of both the power system's initial state and the meteorological evolution between the forecast's initialisation and its target lead time.

It is beyond the scope of the present text to discuss these problems in detail but the key concepts of a 'complex impact' on the power system can be illustrated through a conceptual model, ${ }^{4}$ as shown in Fig. 11.5. 


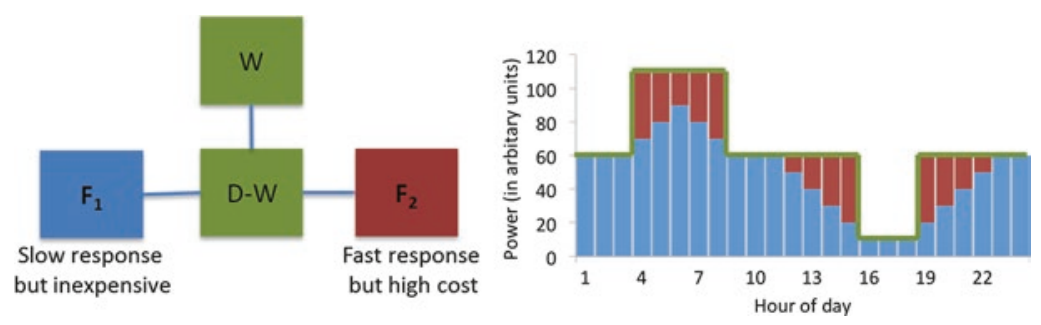

Fig. 11.5 A conceptual model of a simple power system with four components: two fossil fuel generators $\left(F_{1}\right.$ and $\left.F_{2}\right)$ with differing characteristics, wind power generation $W$ and demand $D$. Residual demand $(E=D-W)$, shown by the green line on the time series (right-hand plot), must be met by the combined generation from $F_{1}$ and $F_{2}$. See main text for discussion

Consider a power system with a single demand node, $D$, connected to a wind power source, $W$, the output from which is always instantaneously utilised. As there is no storage of power, the residual demand $E$ (i.e., demand minus wind power, $E=D-W$ ) must be met at all times by two fossil fuel generators, $F_{1}$ and $F_{2} . F_{1}$ has low fuel costs (i.e., it is cheap to generate power with $F_{1}$ ) but changes in its output must occur slowly, whereas $F_{2}$ has high fuel costs (i.e., it is expensive to use) but its output can change rapidly if required.

Consider further a time series of residual demand as shown by the green line on the right hand panel of Fig. 11.5. Initially, the residual demand can be met entirely by $F_{1}$ - the low cost generator-but on hour 4 the residual demand rapidly increases faster than $F_{1}$ can respond and $F_{2}$ must be used to meet the short fall. Crucially, although the residual demand at hour 4 could have been determined using a transfer function applied to the instantaneous meteorological state, ${ }^{5}$ the division of the generation used to meet this residual demand between $F_{1}$ and $F_{2}$ in hour 4 could not have been estimated without also knowing the prior and future meteorological and power system status. In this example, if the residual demand had been higher in hour 3 - and hence $F_{1}(t=3)$ was also higher-then more of the residual demand in hour 4 could have been met with the cheaper $F_{1}$ rather than the more expensive $F_{2}$. In effect, it is not possible to determine the value of $F_{1}$ and $F_{2}$ at a particular point in time (e.g., $t=4$ ) independently of determining $F_{1}$ and $F_{2}$ over many surrounding time steps.

Thus, if one wishes to model the status of the power system at any instant, it is therefore important to correctly represent both the meteorological 
time series trajectory and the power system's time-evolving response to it. It is not sufficient to simply apply a transfer function to an instantaneous 'snapshot' of weather in isolation from the rest of the time series trajectory to produce a full estimate of the power system's status. In practice, the timedependences introduced by power system response constraints are also further complicated by spatial connections introduced by transmission limitations (i.e., finite rates of power transfer between locations). In contrast to 'point forecasting', however, there has been relatively little assessment of 'time-trajectory forecasting' (or spatial patterns of co-dependent meteorological surface variables) in the meteorological research literature in either a weather-forecasting or climate modelling context. Similarly, there has also been relatively little attention paid to the quality of meteorological data used in sophisticated energy system planning and operations studies. New research is, however, beginning to tackle some of these concerns, for example, Bloomfield et al. (2016) highlight that significant errors may arise if insufficiently long weather records are used for power system planning and Pfenninger and Keirstead (2015) have provided a recent example of complex unit commitment modelling in a climate-change context.

Despite the differing levels of energy system impact complexity, many challenges in energy meteorology have similarities to other meteorological applications (e.g., insurance, water and agriculture). The need to calibrate and downscale meteorological variables from coarse prediction datasets to specific localised properties is a particularly ubiquitous problem. Direct meteorological observations of the site (for 'statistical downscaling') and 'dynamical downscaling' (with finer resolution numerical models) can assist in many circumstances, but it is especially challenging when the response of the impacted system depends on more than one meteorological input (in such cases, the co-variability of the meteorological properties may be important as well as the individual meteorological properties themselves). It is also noted that downscaling and calibration only improve the forecast if the large-scale dynamics of the system are well-simulated and, in practice, errors associated with meteorological downscaling and transfer functions are often difficult to separate (see, e.g., Cannon et al. 2017).

\section{SUMMARY}

To summarise, the transfer from 'meteorology' to 'energy' is, in many cases, highly non-linear. This has profound implications for simulation and prediction of energy system impacts, suggesting that forecast skill may be 
strongly influenced by the transformation from meteorological variables to energy variables. In an ideal world, this may act to either increase or decrease the skill of the forecast, depending on the specific characteristics of the forecast problem but, in practice, the skill of an energy forecast will often tend to be lower than the meteorological forecast from which it originates as errors in the transformation process will tend to compound errors in the original meteorology. Careful diagnosis is needed to identify which aspects of the forecasting system - from the meteorological prediction to its downscaling and transformation into an energy property, and finally its conversion into an end-user decision-lead to the dominant sources of error, and to focus analytical resources on the scales and processes where skill is achievable.

\section{Notes}

1. Other examples of similar 'compound impact' problems can be found in peak-load estimation (e.g., Thornton et al. 2017) and simple models for system planning applications (e.g., load duration curves for the estimation of the optimal generation-type mix: Green and Vasilakos 2010; Bloomfield et al. 2016).

2. See, e.g., Staffell and Green (2016) for an introduction to 'merit order' concepts.

3. The residual demand is presented here as total demand minus wind power generation for simplicity. In practice, Lynch (2016) made several additional calculations, removing inflexible generators (such as nuclear) and other varying contributions (such as embedded solar and interconnectors) from the total demand.

4. See, e.g., Wood et al. (2014) and Staffell and Green (2016) for an introduction.

5. That is, a function of the form $E(t=4)=f(\{m(t=4)\})$.

\section{REFERENCES}

Bloomfield, H. C., Brayshaw, D. J., Shaffrey, L. C., Coker, P. J., \& Thornton, H. E. (2016). Quantifying the increasing sensitivity of power systems to climate variability. Environmental Research Letters, 11, 124025.

Brayshaw, D. J., Troccoli, A., Fordham, R., \& Methven, J. (2011). The impact of large scale atmospheric circulation patterns on wind power generation and its potential predictability: A case study over the UK. Renewable Energy, 36, 2087-2096. 
Cannon, D. J., Brayshaw, D. J., Methven, J., Coker, P. J., \& Lenaghan, D. (2015). Using reanalysis data to quantify extreme wind power generation statistics: A 33 year case study in Great Britain. Renewable Energy, 75, 767-778.

Cannon, D. J., Brayshaw, D. J., Methven, J., \& Drew, D. (2017). Determining the bounds of skillful forecast range for probabilistic prediction of system-wide wind power generation. Meteorologische Zeitschrift, 26, 239-252.

Dunning, C. M., Turner, A. G., \& Brayshaw, D. J. (2015). The impact of monsoon intraseasonal variability on renewable power generation in India. Environmental Research Letters, 10, 064002.

Green, R., \& Vasilakos, N. (2010). Market behavior with large amounts of intermittent generation. Energy Policy, 38, 3211-3220.

Lynch, K. J. (2016). Subseasonal forecasting for the energy sector. PhD Thesis, University of Reading.

Lynch, K. J., Brayshaw, D. J., \& Charlton-Perez, A. (2014). Verification of European subseasonal wind speed forecasts. Monthly Weather Review, 142, 2978-2990.

McColl, L., Palin, E. J., Thornton, H. E., Sexton, D. M. H., Betts, R., \& Mylne, K. (2012). Assessing the potential impact of climate change on the UK's electricity network. Climatic Change, 115, 821-835.

Pfenninger, S., \& Keirstead, J. (2015). Renewables, nuclear or fossil fuels? Comparing scenarios for the Great Britain electricity system. Applied Energy, $152,83-93$.

Staffell, I., \& Green, R. (2016). Is there still merit in the merit order stack? The impact of dynamic constraints on optimal plant mix. IEEE Transactions on Power Systems, 31, 43-53.

Taylor, J. W., \& Buizza, R. (2003). Using weather ensemble predictions in electricity demand forecasting. International Journal of Forecasting, 18, 57-70.

Thornton, H. E., Hoskins, B. J., \& Scaife, A. A. (2016). The role of temperature in the variability and extremes of electricity and gas demand in Great Britain. Environmental Research Letters, 11, 114015.

Thornton, H. E., Scaife, A. A., Hoskins, B. J., \& Brayshaw, D. J. (2017). The relationship between wind power, electricity demand and winter weather patterns in Great Britain. Environmental Research Letters, 12, 064017.

Wood, A. J., Wollenberg, B. F., \& Sheble, G. B. (2014). Power generation, operation and control. Hoboken, NJ: Wiley. 632pp. 
Open Access This chapter is distributed under the terms of the Creative Commons Attribution 4.0 International License (http://creativecommons.org/licenses/ by $/ 4.0 /)$, which permits use, duplication, adaptation, distribution and reproduction in any medium or format, as long as you give appropriate credit to the original author(s) and the source, a link is provided to the Creative Commons license and any changes made are indicated.

The images or other third party material in this chapter are included in the work's Creative Commons license, unless indicated otherwise in the credit line; if such material is not included in the work's Creative Commons license and the respective action is not permitted by statutory regulation, users will need to obtain permission from the license holder to duplicate, adapt or reproduce the material. 